\title{
Surgically assisted rapid expansion in two patients with maxillary transverse deficiency
}

\begin{abstract}
The surgeon and orthodontist face daily difficulties with correcting a patient's occlusion Compressed maxilla is a big obstacle to conventional orthodontic treatment or orthognathic surgery. The Surgically Assisted Rapid Palate Expansion is a way to optimize the treatment, based on osteogenic distraction and tissue distraction, reaching more quickly the necessary dimensions of the palate. The purpose of this article is to make the case report of two patients with age differences and show the results obtained in each.
\end{abstract}

Keywords: SARPE, osteogenic distraction, osteogenic distracter
Volume 12 Issue 3 - 2020

\author{
Hugo Romero,' Nadia Irias,' Juan Guifarro,' \\ Alejandro Estrada, ${ }^{3}$ Vima Umanzor, ${ }^{4}$ Fabio \\ Caballero, ${ }^{5}$ Mario Gabrie, ${ }^{5}$ Guillermo \\ Bendaña, ${ }^{5}$ Ishla Mayorga ${ }^{5}$ \\ 'Department of Stomatology, The National Autonomous \\ University of Honduras, Honduras \\ ${ }^{2}$ Department of Maxillofacial Surgery, Hospital Escuela \\ Universitario, Honduras \\ ${ }^{3}$ Department of Orthodontics, Hospital Escuela Universitario, \\ Honduras \\ ${ }^{4}$ Department of Periodontics, The National Autonomous \\ University of Honduras, Honduras \\ ${ }^{5}$ Department of Dental, Hospital Escuela Universitario, \\ Honduras
}

Correspondence: Hugo Romero, Department of Stomatology, The National Autonomous University of Honduras, Honduras, Honduras, Tel 504 33264820,Email cmfalvarenga@gmail.com

Received: February 20, 2020 | Published: May II, 2020
Abbreviations: OME, orthopedic maxillary expansion; MTD, maxillary transversal deficiency

\section{Introduction}

The transverse maxillary discrepancies are a major component of several malocclusion. ${ }^{1}$ The orthopedic and orthodontics forces are used to correct the maxillary transversal deficiency (MTD) in young patients. But in the skeletally mature patient is more challenging because of changes in the osseous articulations of the maxilla with the adjoining bones. ${ }^{2}$

The orthopedic Maxillary Expansion (OME) was first described by Angell over by mid-century XIX. Presently OME has become a routine procedure in the treating maxillary transverse deficiency. The orthodontist and the maxillofacial surgeon should keep in mind that maxillary expansion in mature patients can bring complications later. Such as; Inclination of posterior teeth, root resorption, dental extrusion, periodontal membrane compression, palatal necrosis, periodontal problems. ${ }^{3}$

These procedures are conventionally grouped into 2 categories: segmentation of the maxilla during a Le Fort osteotomy to reposition the individual segments in a widened transverse dimension, and surgically assisted rapid palatal expansion (SARPE); or just SARPE.

The initial procedure is the Le Fort 1 osteotomy; it begins from the anterior nasal spine, moving horizontally above the dental apexes, crossing from the canine fossa, the anterior wall of the maxillary sinus, to the maxillary tuberosity. ${ }^{3}$ In the modification to create the disjunction of the palate, the separation of the intermaxillary suture is performed, until it reaches the end of it in the oral cavity. Subsequently, the distracter is placed without activating it.

Osteogenic distraction is the ability to modify the shape and length of a bone. The different phases of the osteogenic distraction treatment are: ${ }^{4}$

a. Latency phase: Phase where after surgery a clot is formed, which will serve for the new bone apposition. Last 3 to 5 days.

b. Activation phase: Phase where the device is activated gradually, creating tension forces.

c. Consolidation phase: Phase where the bone dimension is the desired one and it is finished mineralizing (Figure $1 \& 2) .^{5}$

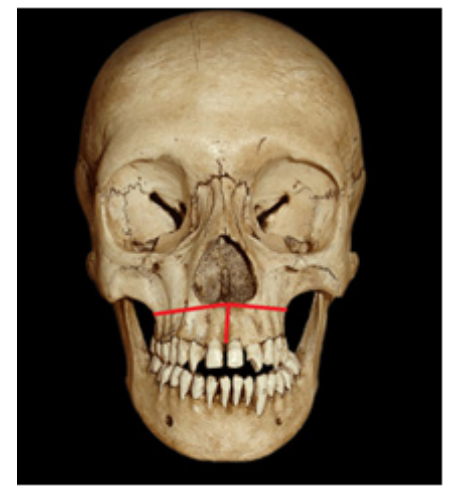

Figure I Image of a Le Fort I osteotomy line is inserted with its modification by performing an osteotomy in the intermaxillary suture. 


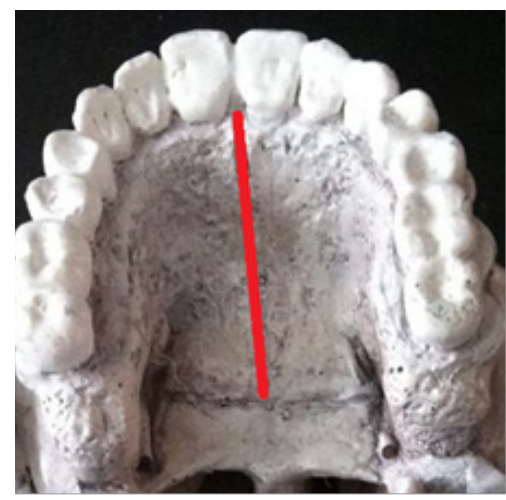

Figure 2 Image showing the osteotomy line in the intermaxillary suture to create disjunction of the palate.

\section{Indications for SARPE}

The following indications have been reported in the literature for SARPE, all applying to a skeletally mature patient with a constricted maxillary arch.

a. To increase maxillary arch perimeter, to correct posterior cross bite, and when no additional surgical jaw movements are planned.

b. Widen the maxillary arch as a preliminary procedure, even if further orthognathic surgery is planned.

c. To provide space for a crowded maxillary dentition when extractions are not indicated.

d. To widen maxillary hypoplasia associated with clefts of the palate.

e. To reduce wide buccal corridors when smiling.

f. To overcome the resistance of the sutures when OME has failed.

\section{Diagnosis}

Several methods used to diagnose this condition. Clinical evaluation, model analysis, occlusogram and radiographic measurements have been recommended. For an accurate evaluation. The clinical evaluation includes the evaluation of the maxillary shape and symmetry of the arch, the shape of the palate, the width of the buccal corridors when smiling, occlusion, and the predominant mode of breathing. ${ }^{6}$

\section{Case report}

The report made in the surgical database of the Hospital Escuela Universitario of Honduras, two patients undergoing surgically assisted palatal expansion with skeletal facial problems, in the transverse axis of the upper jaw with diagnoses of:

a. Class III of maxillary angle hypo development.

b. Bite crosses with maxillary / canine constriction included.

In both patients the same surgical technique was performed. Using the Hyrax as a distracter, anchoring the device to the first premolars and the first molars by orthodontic bands. Activation began on the sixth postoperative day and was carried out in an activation period by turning 2 times a day for 12 days. These procedures were performed on an outpatient basis with minimal morbidity, under general anesthesia with similar periods of time during activation in $\mathrm{mm}$. of the hyrax, obtaining different symptoms and results by carrying out (Figure 3) ${ }^{7,8}$

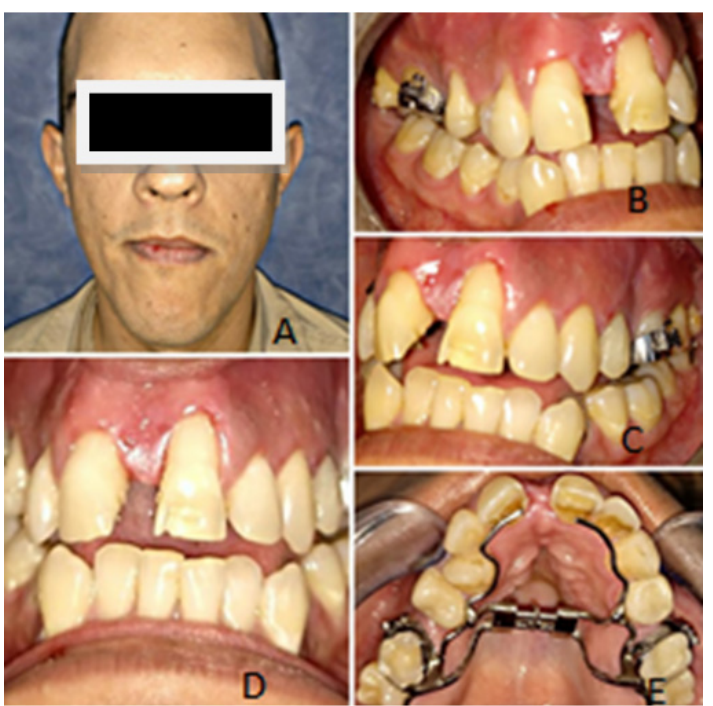

Figure 3 Extra and intraoral photographs showing the initial condition of the patient and his distracting Hyrax type: See Maxillary Convergence.A,D: Extra and oral Frontal photo; B, C: Lateral Oral Photo; E: Photo with the Hyrax.

The same surgical procedure. Type of palatal division pattern: disjunction of the middle palatal suture.

Type I: pattern of palatal division: complete disjunction of the middle palatal suture of the nasal spine anterior to the posterior nasal spine.

Type II: incomplete disjunction of the middle palatal suture, from the anterior nasal spine to the palatal transverse suture, with little or no posterior division and therefore not including the palatine bone (Figure 4). ${ }^{9}$

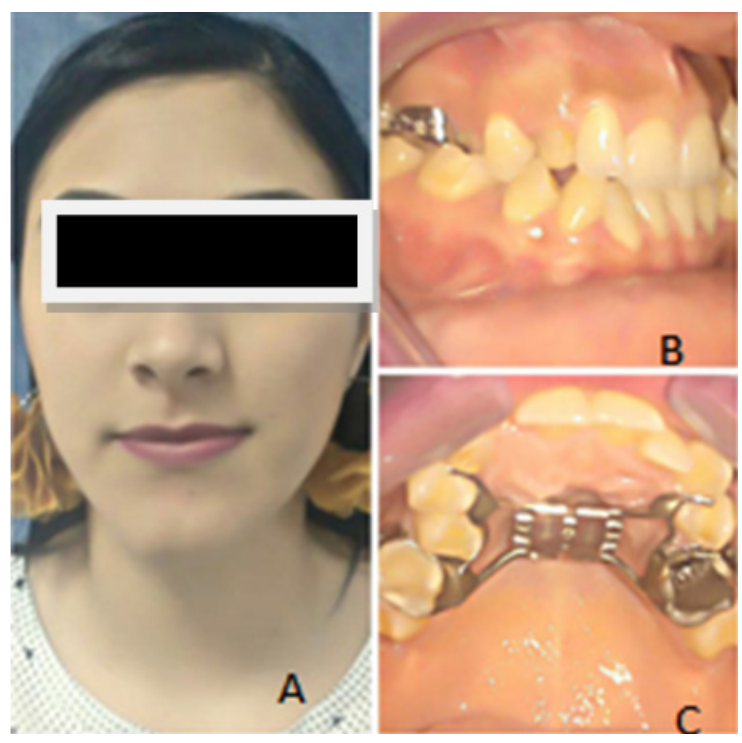

Figure 4 Patient with maxillary constriction, creating a pseudo class III. Undergoing the same procedure.A: frontal photo; B: lateral oral photo; C: oral photo with the hyrax.

Transverse maxillary deficiency is a dentofacial deformity clinically characterized by posterior unilateral or bilateral cross bite 8.1 , dental crowding, excessive lingual inclination of the posterior teeth, a triangular dental arch, deep palate, and predominant mouth breathing can cause maxillary growth deficiency. Among the 
complications is septal deviation, asymmetric distraction, necrosis of the incisors, dental mobility, infections, dental coloration, loss of periodontal bone.

While it is true that rapid expansion of the surgically assisted palate can lead to complications in adult patients, it is a procedure that has proven to have good results in young patients. SARPE is a fairly safe procedure that allows transversal coordination of dental arches without tooth extraction when MTD is greater than $4 \mathrm{~mm}$. This quantity of $4 \mathrm{~mm}$ is, for us, the limit between the correction of MTD in a single operation by means of a segmented osteotomy of Le Fort I and the need for a SARPE before sagittal discrepancy correction. MTD often goes along with maxillary retrognathism and is therefore, a frequent feature in class III malocclusion (Figure 5). ${ }^{10-14}$

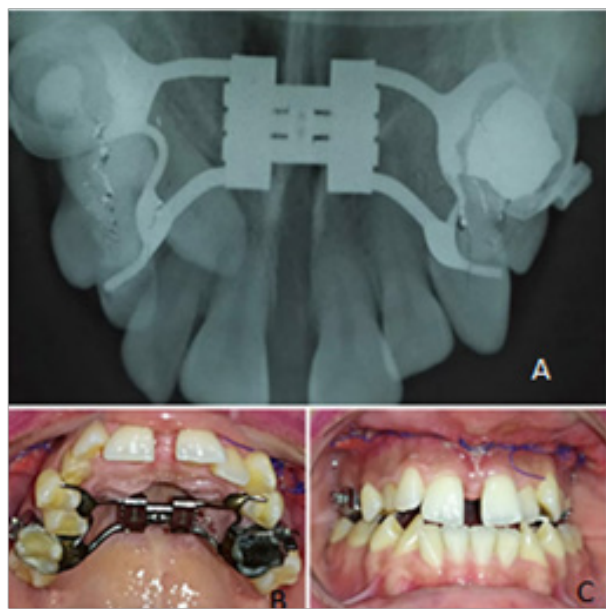

Figure $5 \mathrm{~A}$ : Occlusal radiography showing the expansion of the palate and the disjunction of the intermaxillary suture; B: oral photography showing hyrax. C: Fontal photography where the expansion of the archway is evidenced.

\section{Discussion}

MTD is best determined in dental molds through inter-relationship analysis. Classically it can be managed in three ways: pure orthodontics treatment, surgery "once" (segmented osteotomy Le Fort 1) or Surgery 'Two-step surgery' (SARPE first followed by a Le Fort 1 piece (second operation). The indications for correct orthodontic correction of MTD depend closely on the degree of ossification of the palatal suture. Different There are methods for estimating the ossification of sutures. The exam is inapplicable in vivo. Radiological exam (2D and 3D) is not reliable. The patient's age remains the usual criteria. ${ }^{15,16}$

For many authors, ossification is usually considered complete at an age between 14 and 18 years. After this period, orthodontic treatment is random, especially if necessary the expansion exceeds $4 \mathrm{~mm}$, the risks are an insufficient result, dental versions and root extrusions through the vestibular cortex bone. Already in 1972, Steinhauser reported a 2-piece Le Fort I osteotomy technique that associates a classic osteotomy of Le Fort I and a surgical division of the palate over the midline. This has now become a well-established procedure, commonly used to correct maxillary transverse discrepancies in adults. ${ }^{17-19}$

Segmental osteotomy is the preferred option for the correction of MTD at least 5 to $6 \mathrm{~mm}$. However, this is often associated with postsurgical instability and relapse. Infection, oral fistulas and damage to adjacent teeth are commonly reported complications. SARPE may be indicated in cases where pure orthodontics expansion failed or is insufficient or when MTD exceeds 4 to $6 \mathrm{~mm}$.

\section{Conclusion}

In this case report, SARPE was performed in two patients, with different age types. In both patients the results were different, with age being the factor that intervened in both results, where the older patient had complications. This technique is used when the amount of expansion that is needed is more than the tissues withstand, thus producing, in turn, aosteogenic distraction.

\section{Acknowledgments}

None.

\section{Conflicts of interest}

The authors declare there are no conflicts of interest.

\section{Funding}

None.

\section{References}

1. Suri L, Taneja P. Surgically assisted rapid palatal expansion: A literature review. Am J Orthod Dentofacial Orthop. 2008;133(2):290-302.

2. Angell EH. Treatment of irregularity of permanent adult teeth. Dent Cosmos. 1860;1:540

3. Timms DJ. Emerson C. Angell (1822-1903). Founding father of rapid maxillary expansion. Dent Hist. 1997;(32):3-12.

4. Hupp James. Contemporary Maxillofacial Surgery. 6 ed. Elsevier: Barcelona, España; 2014:720

5. Barrabé A, Meyer C, Bonomi H, et al. Surgically assisted rapid palatal expansion in class III malocclusion: Our experience. J Stomatol Oral Maxillofac Surg. 2018;119(5):384-388.

6. Liu P, Chen H, Shi X, et al. Conservative treatment of a young adult patient with a moderate skeletal Class III malocclusion by applying the temporary anchorage devices and the surgically assisted rapid palatal expansion. Clin Case Rep. 2017;5(12):2003-2011.

7. Chamberland S, Proffit WR. Short-term and long-term stability of surgically assisted rapid palatal expansion revisited. Am J Orthod Dentofacial Orthop. 2011;139(6):815-822.

8. Behnia H, Mohammad-Rahimi H, Behnaz M. Treatment of an Adult Skeletal Class III Patient with Surgically Assisted Rapid Palatal Expansion and Facemask. Case Rep Dent. 2019;2019:8251903.

9. Pereira MD, Koga AF, Prado GPR, et al. Complications From Surgically Assisted Rapid Maxillary Expansion With HAAS and HYRAX Expanders. J Craniofac Surg. 2018;29(2):275-278.

10. Rômulo de Medeiros J, Ferraro Bezerra M, Gurgel Costa FW, et al Does pterygomaxillary disjunction in surgically assisted rapid maxillary expansion influence upper airway volume? A prospective study using Dolphin Imaging 3D. Int J Oral Maxillofac Surg. 2017;46(9):1094-1101.

11. Moura LB, Spin-Neto R, Sverzut CE, et al. Evaluation of the palatal split pattern in surgically rapid maxillary expansion-comparison of two techniques. Oral Maxillofac Surg. 2016;20(3):255-258.

12. Dahiya S, Chitra P, Rao SS, et al. Modified SARME (Surgically Assisted Rapid Maxillary Expansion) in Conjunction with Orthodontic TreatmentA Case Report. J Clin Diagn Res. 2015;9(10):ZD20-ZD22. 
13. Haas AJ. The treatment of maxillary deficiency by opening the midpalatal suture. Angle Orthod. 1965;35:200-217.

14. Timms DJ. A study of basal movement with rapid maxillary expansion. Am J Orthod. 1980;77(5):500-507.

15. Wertz RA. Skeletal and dental changes accompanying rapid midpalatal suture opening. Am J Orthod. 1970;58(1):41-66.

16. Isaacson RJ, Murphy TD. Some effects of rapid maxillary expansion in cleft lip and palate patients. Angle Orthod. 1964;34(3):143-154.
17. Mommaerts MY. Transpalatal distraction as a method of maxillary expansion. Br J Oral Maxillofac Surg. 1999;37(4):268-272.

18. Zimring JF, Isaacson RJ. Forces produced by rapid maxillary expansion. 3. Forces present during retention. Angle Orthod. 1965;35:178-186.

19. Barber AF, Sims MR. Rapid maxillary expansion and external root resorption in man: a scanning electron microscope study. Am J Orthod. 1981;79(6):630-652. 